\title{
Clinical prediction score for diagnosing non-diabetic renal disease in patients with type 2 diabetes mellitus: a cohort study
}

\section{Ekkapong Surinrat}

Thammasat University Faculty of Medicine

Thanee Eiamsitrakoon

Thammasat University Faculty of Medicine

Pajaree Krisanapan

Thammasat University Faculty of Medicine

Aphichat Chatkrailert

Thammasat University Faculty of Medicine

Anake Yoosabai

Thammasat University Faculty of Medicine

\section{Opas Traitanon}

Thammasat University Faculty of Medicine

\section{Adis Tasanarong}

Thammasat University Faculty of Medicine

\section{Mongkon Charoenpitakchai}

Phramongkutklao College of Medicine

Pichaya Tantiyavarong ( $\sim$ pichaya_t@tu.ac.th )

Thammasat University Faculty of Medicine https://orcid.org/0000-0003-0793-794X

\section{Research article}

Keywords: Non-diabetic renal disease, diabetic nephropathy, clinical prediction score, kidney biopsy, diagnosis, type 2 diabetes mellitus

Posted Date: July 20th, 2020

DOI: https://doi.org/10.21203/rs.3.rs-41394/v1

License: (c) (1) This work is licensed under a Creative Commons Attribution 4.0 International License. Read Full License 


\section{Abstract}

Background: When non-diabetic renal disease (NDRD) is suspected, kidney biopsy is used for definite diagnosis; however, this is not always easily available and may lead to complications. A clinical prediction score may help selecting appropriate patients for kidney biopsy.

Methods: A retrospective cohort study was conducted in type 2 diabetes mellitus (T2DM) patients with atypical features of diabetic nephropathy (DN), who had kidney biopsy at Thammasat University Hospital from 2011-2019. We divided patients into NDRD alone, coexisting NDRD and DN, and DN alone, confirmed by pathological diagnosis. We developed a clinical prediction score by weighing coefficients of predictors in a multivariable logistic model. Internal validation was performed with bootstrapping.

Results: We included 81 patients: 28 (34\%) had NDRD alone, 15 (18\%) had coexisting NDRD and DN, and $38(41 \%)$ had DN alone. Primary membranous nephropathy, primary focal and segmental glomerulosclerosis (FSGS), and secondary FSGS were prevalent in any NDRD. Absence of diabetic retinopathy (DR) showed a significant association with any NDRD (OR 3.72; 95\% Cl, 1.28-10.8; $p=0.02$ ). The prediction score, AUC of $0.75(95 \% \mathrm{Cl}, 0.63-0.86)$, had four predictors: duration of $\mathrm{DM}<10$ years, eGFR $>30 \mathrm{ml} / \mathrm{min} / 1.73 \mathrm{~m}^{2}, \mathrm{HbA} 1 \mathrm{c}<8 \%$, and absence of DR. Higher scores were associated with higher probability of NDRD.

Conclusions: This clinical prediction score appears to be a useful tool to determine NDRD probability. T2DM patients with atypical presentation of DN with lower scores (0-2) may defer kidney biopsy.

\section{Background}

Diagnosing non-diabetic renal disease (NDRD) in patients with type 2 diabetes mellitus (T2DM) is often problematic and typically requires a kidney biopsy for definite diagnosis. The common pathological diagnoses of NDRD are membranous nephropathy (MN), focal segmental glomerulosclerosis (FSGS) and IgA nephropathy [1-3]. Unfortunately, it remains difficult to diagnose as NDRD may present alone or in combination with diabetic nephropathy (DN); the latter obscures the classical presentation of each disease. Thus, kidney biopsy is the standard investigation for definite diagnosis [4]. However, this invasive procedure may have bleeding complications and its availability is limited in some resourceconstrained hospitals.

The decision to perform a kidney biopsy depends on the likelihood of NDRD. According to the Kidney Disease Outcomes Quality Initiative (KDOQI) guideline [4], patients with atypical presentations of DN with the absence of diabetic retinopathy (DR), low or rapidly declining glomerular filtration rate (GFR), rapidly increasing proteinuria or nephrotic syndrome, the presence of active urinary sediment, or signs and symptoms of systemic disease, should be evaluated for NDRD. Even using these criteria, NDRD prevalence was found in only half of some biopsy reports [1,5-7]. It appears there is a knowledge gap toward improving NDRD diagnostic performance. 
Previous studies have only reported predictors, such as absence of DR, duration of diabetic mellitus (DM), degrees of proteinuria, Hemoglobin A1c ( $\mathrm{HbA1c}$ ), or levels of creatinine or GFR associated with NDRD, but none have mentioned the utility of a combined predictive probability [3, 7-9]. A clinical prediction score combines predictors in the model, informs clinicians and patients about disease probability, and can aid in decision-making [10]. In this study, we aimed to develop a simplified clinical prediction score for NDRD to help determine the appropriate clinical setting for kidney biopsy in T2DM with atypical presentation.

\section{Methods}

Data were collected by retrospective chart review; we included all patients with T2DM who had undergone kidney biopsy ${ }^{3} 18$ years old at Thammasat University Hospital between January 2011 and December 2019. The diagnosis of T2DM was obtained from history and criteria established by the American Diabetes Association [11]. We excluded patients with history of kidney transplantation and inadequate specimen for interpreting pathological diagnosis from kidney biopsy. The study was approved by the Human Research Ethics Committee of Thammasat University No 1 (Faculty of Medicine), certificate of approval 123/2020.

We collected all clinical parameters including age, gender, body mass index (BMI), duration of DM, presence of DR, hypertension, established cardiovascular diseases (CVD), also indications for kidney biopsy. The presence of DR was examined and recorded by an ophthalmologist. The duration of DM refers to the time from first diagnosis to kidney biopsy. Hypertension was defined as either having a recorded past history of hypertension or systolic blood pressure $\geq 140 \mathrm{mmHg}$ and/or diastolic blood pressure $\geq 90 \mathrm{mmHg}$, determined at clinical evaluation for kidney biopsy in the absence of any antihypertensive drug. CVD was a past history of myocardial infarction or congestive heart failure in previous medical records.

Laboratory results were collected at the time of biopsy including complete blood count (CBC), blood urea nitrogen (BUN), serum creatinine using enzymatic method with estimated glomerular filtration rate (eGFR) using the Chronic Kidney Disease Epidemiology Collaboration (CKD-EPI) [12], urinary analysis, urine protein creatinine ratio (UPCR), fasting plasma glucose (FPG), and HbA1c. Hematuria was defined as a red blood cell (RBC) count of $\geq 3$ cells in urine examination. UPCR was calculated by dividing urine protein $(\mathrm{mg} / \mathrm{dL})$ by urine creatinine $(\mathrm{mg} / \mathrm{dL})$.

All kidney specimens were examined using light microscope and immunofluorescence assay. Because our hospital does not routinely perform electron microscope, very few cases had these results. DN diagnosis and classification were made using criteria established by the Renal Pathology Society in 2010 [13]. The pathological diagnosis was divided into three groups consisting of DN, NDRD, and coexisting DN and NDRD (DN+NDRD).

\section{Statistical analysis}


All statistical analyses were performed using Stata software, v16.0 (StataCorp). All $p$ values were twosided; $p$ value $<0.05$ was considered statistically significant. Continuous variables data were presented as mean \pm standard deviation (SD) or median and interquartile range (IQR). Categorical variables were expressed as frequency and percentages.

In the processes of model specification, we reviewed literature, which reported predictors of NDRD, and explored associations with any type of NDRD (NDRD alone or DN+NDRD) in univariable logistic model. Any predictors, in which $p$ value $<0.2$ in univariable analyses, were selected for multivariable logistic regression. Then, we tested the performance of the final model with Hosmer-Lemeshow test and area under receiver operating characteristic curve (AUC).

To generate the clinical prediction score, coefficients of all predictors were weighed by dividing the lowest coefficient and rounded into integers. The scores of individual patients were matched with the estimated probability of it being NDRD in the final model, also referred to as the developed model. Next, these individual scores were tested for predictive performance using AUC.

We performed internal validation using a bootstrapping procedure with a 1,000-bootstrap sample. This procedure quantified the optimism of the developed model. We revised the new model, now called the optimism-adjusted model using the uniform shrinkage factor. Then, the scores of individual patients were matched again with estimated probability in this optimism-adjusted model. AUC was again tested.

For clinical implications, we now proposed an algorithm by categorizing the clinical prediction scores into three groups: low probability of NDRD, intermediate probability, and high probability with our suggestions for kidney biopsy decision.

\section{Results}

A total of 81 T2DM patients, who had kidney biopsies, were included. Indications of kidney biopsy were the sudden onset of proteinuria or nephrotic syndrome (39.5\%), rapidly decreasing eGFR (37\%), AGN or RPGN (12.4\%), and others (11\%). Of the 81 patients, 38 (46.9\%) were DN alone, 28 (34.6\%) were NDRD alone, and $15(18.5 \%)$ were DN+NDRD.

The baseline clinical characteristics of all patients, as well as DN, NDRD, and DN+NDRD groups, are shown in Table 1. Mean age of all patients was $56.0 \pm 13.1$ years. The amount of males and females were proportional. Median duration of DM was highest in the DN group being 10 years, with an average of five years for both NDRD and DN+NDRD group. Presence of DR was predominantly high in the DN group, accounting for $63 \%$ of those cases, with $53 \%$ having DN+NDRD, and $25 \%$ NDRD. Mean eGFR was lowest in the DN group as $31.6 \mathrm{ml} / \mathrm{min} / 1.73 \mathrm{~m}^{2}$, with $40.6 \mathrm{ml} / \mathrm{min} / 1.73 \mathrm{~m}^{2}$ in DN+NDRD, and 42 $\mathrm{ml} / \mathrm{min} / 1.73 \mathrm{~m}^{2}$ in NDRD. Hematuria was found in high proportion in all three groups, but urine RBC $>30$ 
cells/HPF predominated in the NDRD group. Mean HbA1c was $7.8 \%$ for the DN group, with $7.4 \%$ in the DN+NDRD group, and $6.9 \%$ in NDRD group.

\section{Pathological findings of NDRD}

Forty-three patients were diagnosed with some type of NDRD: 28 NDRD alone and 15 DN+NDRD (Table 2). Primary membranous nephropathy, primary focal segmental glomerulosclerosis and secondary focal segmental glomerulosclerosis were the three most common lesions, each accounting for $14 \%$. Pauciimmune glomerulonephritis, IgA nephropathy, and postinfectious glomerulonephritis were also prevalent in these groups.

\section{Predictors associated with NDRD}

Univariable and multivariable binary logistic models were used to define associations for predictors and any type of NDRD (NDRD alone or DN+NDRD). As mentioned before, four predictors (duration of DM $<10$ years, eGFR $>30 \mathrm{ml} / \mathrm{min} / 1.73 \mathrm{~m}^{2}, \mathrm{HbA} 1 \mathrm{c}<8 \%$, and absence of $\mathrm{DR}$ ) were selected for the multivariable model due to their $p$ value being $<0.2$ in the univariable model. The absence of DR showed a significant association with NDRD with the highest magnitude of association (OR 3.72; 95\% Cl, 1.28-10.8; $p=0.02$ ), which meant a higher likelihood of NDRD. Other predictors, which demonstrated clinically significant magnitudes of association, but without statistical significance, were duration of $D M<10$ years (OR 1.50; $95 \% \mathrm{Cl}, 0.52-4.35 ; p=0.46)$, eGFR $>30 \mathrm{ml} / \mathrm{min} / 1.73 \mathrm{~m}^{2}$ (OR 2.31; $\left.95 \% \mathrm{Cl}, 0.73-7.34 ; p=0.16\right)$, and $\mathrm{HbA} 1 \mathrm{c}<8 \%$ (OR 2.82; 95\% Cl, 0.79-10.0; $p=0.11$ ) (Table 3). The Hosmer-Lemeshow test was performed to test goodness of fit and demonstrated a $p$ value of 0.29 . The AUC of the model was $0.75(95 \% \mathrm{Cl}, 0.64-0.86)$.

\section{Clinical prediction score}

Within our multivariable model, the linear equation was: logodds (NDRD) $=-1.75+0.41$ (DM duration $<10$ years $)+0.84\left(\right.$ eGFR $\left.>30 \mathrm{ml} / \mathrm{min} / 1.73 \mathrm{~m}^{2}\right)+1.03(\mathrm{HbA} 1 \mathrm{c}<8 \%)+1.31$ (absence of DR). In here, we used the lowest coefficients of 0.41 as a denominator, and the weighted scores were assigned as 1 for DM duration $<10$ years, 2 for eGFR $>30 \mathrm{ml} / \mathrm{min} / 1.73 \mathrm{~m}^{2}$ and $\mathrm{HbA} 1 \mathrm{c}<8 \%$, and 3 for absence of DR.

In Table 4, we compared the estimated probability of being NDRD from the developed and optimismadjusted models. The score ranged from 0 to 8 , with a higher score associated with greater probability of NDRD. The score was divided into three categories: low probability of NDRD (score 0-2), intermediate probability (score 3-5) and high probability (score 6-8) (Figure 1). The clinical prediction score had an AUC of 0.75 in the developed model and 0.70 in the optimism-adjusted model.

\section{Discussion}

This study aimed to develop a tool for diagnosing NDRD in T2DM patients with atypical presentations of DN. We found some predictors that indicated when it was more likely for NDRD to occur, such as duration 
of DM, eGFR, HbA1c, and absence of DR. Clinical predictive scores from these can be used as a risk stratification tool whether kidney biopsy should be performed or not.

The majority of our cases had kidney biopsies due to sudden onset of proteinuria or nephrotic syndrome or rapid eGFR decline; this conformed with standard indications in the KDOQI guideline [4]. Unfortunately, we found only around half (53\%) of the study population had any type of NDRD (NDRD alone or DN+NDRD), and the remainder had DN alone. This may mean the kidney biopsy was unnecessarily performed in those cases and atypical features can be found in DN. Previous kidney biopsy studies reported varying prevalence of NDRD. In a literature review, Kanodia et al [1], found the percentage of NDRD ranged from 45-75\%. Sharma et al [3] reviewed 620 biopsies in patients with diabetes and noted $63 \%$ had NDRD. Because of this moderate yield in NDRD detection, there is a room for a diagnostic tool to differentiate T2DM patients with low or high probability of NDRD.

Previous research has reported some predictors are associated with NDRD. Similar to our findings, the absence of DR was the strongest predictor of NDRD in many studies $[2,5,6,8,9,14,15]$. In other words, when DR is present, it is a suggestive of DN in T2DM, because both are microvascular complications. Longer duration of DM is inversely associated with NDRD. Dong et al [8] found DM history $\leq 5$ years had OR of $4.6(95 \% \mathrm{Cl}, 1.7-12.5)$, Kritmetapak et al [7] reported a duration of $\mathrm{DM}>8$ years with an $\mathrm{OR}$ of 0.15 (95\% Cl, 0.04-0.49), and finally, Yang et al[9] showed a duration of DM $<10$ and $>5$ years had OR of 0.06 $(95 \% \mathrm{Cl}, 0.97-0.75)$. In our opinion, the exact duration of T2DM seems difficult to obtain. Therefore, we used a cutoff of $<10$ years for simplicity and found significant associations in the univariable model but not within the multivariable model: this latter phenomenon may be due to low power. Higher renal function was found to be a significant predictor in a recent study [9], showing eGFR $\geq 90 \mathrm{ml} / \mathrm{min} / 1.73 \mathrm{~m}^{2}$ had OR of 6.38 (95\% Cl, 1.58-25.7). High HbA1c or fasting blood sugar were reported in a few studies [5, 7]. Unlike previous reports $[6,9,15]$, we could not find any association of proteinuria levels with NDRD as the majority of our population already had macroalbuminuria.

This study may be the first to combine predictors into a comprehensive risk stratification score. For clinical purpose and ease of use, we divided these scores into low, intermediate and high probabilities of NDRD. We have also proposed an algorithm of care for each category, especially in the low probability of NDRD group, where patients may defer kidney biopsy (Figure 1). This could be useful in settings with limited resources for kidney biopsy and prevent patient discomfort and surgical complications. However, with intermediate and high probability, a kidney biopsy is still suggested for definite diagnosis of NDRD.

We must still point out that there are some essential limitations to consider in our work here. First, the small sample size is likely to have affected the statistical power, potentially creating a risk of overfitting and optimism bias. We attempted to manage this by choosing predictors from previous studies and using a $p$ value of 0.20 to select predictors into the multivariable analysis, without backward elimination if the predictors were not statistically significant. Internal validation was done with bootstrapping to adjust the developed model for optimism. Second, the inherent nature of retrospective data collection can 
affect data quality in the verification of outcomes or missingness. As around $5 \%$ of our data was missing for the essential predictors, complete-case analysis was used.

\section{Conclusions}

A clinical prediction score for NDRD is a useful risk stratification tool for kidney biopsy in T2DM patients with atypical presentations. Using multiple predictors as opposed to a sole one appeared to improve the predictive ability; hopefully, this kind of score can lead to the deferment of unnecessary kidney biopsy. It would be interesting to apply this prediction score in other populations at other centers to observe if we can ameliorate its external validity.

\section{Declarations}

\section{Ethics approval and consent to participate}

The study was approved by the Human Research Ethics Committee of Thammasat University No 1 (Faculty of Medicine), certificate of approval 123/2020.

\section{Consent for publication}

Not applicable.

\section{Availability of data and materials}

The datasets used and/or analyzed during the current study are available from the corresponding author on reasonable request.

\section{Competing interests}

The authors declare that they have no competing interests

\section{Funding}

Not applicable.

\section{Authors's contributions}

E.S. and P.T. designed the study. E.S. collected data. P.T. and Th.E. analyzed the data and made the figure. All the authors interpreted and gave suggestions for additional analyses. E.S. and P.T. drafted the manuscript. All the authors revised the manuscript and approved the final version of the manuscript.

\section{Acknowledgements}

We would like to thank all patients for contributing data via hospital records. This assignment could not have been completed without the help of the Dialysis Unit at the Kittiwattana Building for providing us 
with useful data regarding kidney biopsies and other information. We also thank Debra Kim Liwiski for the English editing.

\section{Authors' information}

Not applicable.

\section{Abbreviations}

AUC: area under receiver operating characteristic curve

BMI: body mass index

BUN: blood urea nitrogen

CBC: complete blood count

CKD-EPI: Chronic Kidney Disease Epidemiology Collaboration

CVD: cardiovascular diseases

DM: diabetic mellitus

DN: diabetic nephropathy

DR: diabetic retinopathy

eGFR: estimated glomerular filtration rate

FPG: fasting plasma glucose

FSGS: primary focal and segmental glomerulosclerosis

GFR: glomerular filtration rate

HbA1c: Hemoglobin A1c

IQR: interquartile range

KDOQI: Kidney Disease Outcomes Quality Initiative

MN: membranous nephropathy

NDRD: non-diabetic renal disease

SD: standard deviation 
T2DM: type 2 diabetes mellitus

UPCR: urine protein creatinine ratio

RBC: red blood cell

\section{References}

1. Kanodia KV, Vanikar AV, Nigam L, Patel RD, Suthar KS, Patel H: Clinicopathological study of nondiabetic renal disease in type 2 diabetic patients: A single center experience from India. Saudi J Kidney Dis Transp/ 2017, 28(6):1330-1337.

2. Oh SW, Kim S, Na KY, Chae DW, Kim S, Jin DC, Chin HJ: Clinical implications of pathologic diagnosis and classification for diabetic nephropathy. Diabetes Res Clin Pract 2012, 97(3):418-424.

3. Sharma SG, Bomback AS, Radhakrishnan J, Herlitz LC, Stokes MB, Markowitz GS, D'Agati VD: The modern spectrum of renal biopsy findings in patients with diabetes. Clin J Am Soc Nephrol 2013, 8(10):1718-1724.

4. Kdoqi: KDOQI Clinical Practice Guidelines and Clinical Practice Recommendations for Diabetes and Chronic Kidney Disease. Am J Kidney Dis 2007, 49(2 Suppl 2):S12-154.

5. Mou S, Wang Q, Liu J, Che X, Zhang M, Cao L, Zhou W, Ni Z: Prevalence of non-diabetic renal disease in patients with type 2 diabetes. Diabetes Res Clin Pract 2010, 87(3):354-359.

6. Yenigun EC, Dede F, Ozturk R, Turgut D, Koc E, Piskinpasa SV, Ozkayar N, Odabas AR: Non-Diabetic renal disease in Diabetes Mellitus: clinical features and renal biopsy findings. Hippokratia 2015, 19(2):148-152.

7. Kritmetapak K, Anutrakulchai S, Pongchaiyakul C, Puapairoj A: Clinical and pathological characteristics of non-diabetic renal disease in type 2 diabetes patients. Clin Kidney J 2018, 11(3):342-347.

8. Dong Z, Wang Y, Qiu Q, Zhang X, Zhang L, Wu J, Wei R, Zhu H, Cai G, Sun X et al: Clinical predictors differentiating non-diabetic renal diseases from diabetic nephropathy in a large population of type 2 diabetes patients. Diabetes Res Clin Pract 2016, 121:112-118.

9. Yang Z, Feng L, Huang Y, Xia N: A Differential Diagnosis Model For Diabetic Nephropathy And NonDiabetic Renal Disease In Patients With Type 2 Diabetes Complicated With Chronic Kidney Disease. Diabetes Metab Syndr Obes 2019, 12:1963-1972.

10. Collins GS, Reitsma JB, Altman DG, Moons KG: Transparent reporting of a multivariable prediction model for individual prognosis or diagnosis (TRIPOD): the TRIPOD statement. BMJ 2015, 350:g7594.

11. American Diabetes A: 2. Classification and Diagnosis of Diabetes: Standards of Medical Care in Diabetes-2019. Diabetes Care 2019, 42(Suppl 1):S13-S28.

12. Levey AS, Stevens LA, Schmid CH, Zhang Y, Castro AF, Feldman HI, Kusek JW, Eggers P, Van Lente F, Greene T et al: A New Equation to Estimate Glomerular Filtration Rate. Annals of Internal Medicine 2009, 150(9):604. 
13. Tervaert TW, Mooyaart AL, Amann K, Cohen AH, Cook HT, Drachenberg CB, Ferrario F, Fogo AB, Haas M, de Heer E et al: Pathologic classification of diabetic nephropathy. J Am Soc Nephrol 2010, 21(4):556-563.

14. Soleymanian T, Hamid G, Arefi M, Najafi I, Ganji MR, Amini M, Hakemi M, Tehrani MR, Larijani B: Nondiabetic renal disease with or without diabetic nephropathy in type 2 diabetes: clinical predictors and outcome. Ren Fail 2015, 37(4):572-575.

15. Bermejo S, Soler MJ, Gimeno J, Barrios C, Rodríguez E, Mojal S, Pascual J: Predictive factors for nondiabetic nephropathy in diabetic patients. The utility of renal biopsy. Nefrología (English Edition) 2016, 36(5):535-544.

\section{Tables}

\section{Table 1 Baseline characteristics}




\begin{tabular}{|c|c|c|c|c|}
\hline Parameter & $\begin{array}{c}\text { Total } \\
(\mathrm{n}=81)\end{array}$ & $\begin{array}{c}\text { DN } \\
(n=38)\end{array}$ & $\begin{array}{l}\text { NDRD } \\
(n=28)\end{array}$ & $\begin{array}{c}\text { DN+NDRD } \\
(n=15)\end{array}$ \\
\hline Age, year & $56.0 \pm 13.1$ & $54.1 \pm 12.7$ & $58.4 \pm 14.2$ & $56.3 \pm 12.2$ \\
\hline Male & $41(51)$ & $19(50)$ & $13(46)$ & $9(60)$ \\
\hline BMI, $\mathrm{kg} / \mathrm{m}^{2}$ & $25.6 \pm 5.5$ & $25.2 \pm 4.6$ & $25.5 \pm 6.5$ & $26.8 \pm 5.5$ \\
\hline Duration of DM, years & $8[4,12]$ & $10[6,12]$ & $5[3,10]$ & $5,[3,10]$ \\
\hline Duration $>10$ years & $37(46)$ & $22(58)$ & $10(36)$ & $5(33)$ \\
\hline Presence of DR & $39(48)$ & $24(63)$ & $7(25)$ & $8(53)$ \\
\hline Hypertension & $71(88)$ & 34 (89) & $23(82)$ & $14(93)$ \\
\hline Established CVD & $11(14)$ & $6(16)$ & $2(7)$ & $3(20)$ \\
\hline Serum creatinine, mg/dL & $2.7 \pm 1.6$ & $2.9 \pm 1.7$ & $2.5 \pm 1.7$ & $2.3 \pm 1.3$ \\
\hline $\mathrm{eGFR}, \mathrm{ml} / \mathrm{min} / 1.73 \mathrm{~m}^{2}$ & $37.0(29.0)$ & $31.6(24.9)$ & $42.3(34.5)$ & $40.6(26.8)$ \\
\hline \multicolumn{5}{|l|}{ eGFR category } \\
\hline$>60$ & $17(21)$ & $5(13)$ & $8(29)$ & $4(27)$ \\
\hline $30-60$ & $20(25)$ & $9(24)$ & $6(21)$ & $5(33)$ \\
\hline$<30$ & $44(54)$ & $24(63)$ & $14(50)$ & $6(40)$ \\
\hline $\mathrm{Hb}, \mathrm{g} / \mathrm{dL}$ & $10.7 \pm 2.3$ & $10.5 \pm 1.8$ & $11.0 \pm 2.9$ & $10.8 \pm 2.4$ \\
\hline Hematuria & $59(73)$ & 30 (79) & $18(64)$ & $11(73)$ \\
\hline \multicolumn{5}{|l|}{ Urine RBC, cell/HPF } \\
\hline $0-3$ & $22(27)$ & $8(21)$ & $10(36)$ & $4(27)$ \\
\hline $3-30$ & $42(59)$ & $24(63)$ & $9(32)$ & $9(60)$ \\
\hline$>30$ & $17(21)$ & $6(16)$ & $9(32)$ & $2(13)$ \\
\hline UPCR, mg/g & $8.3 \pm 6.7$ & $9.2 \pm 7.0$ & $7.0 \pm 7.0$ & $8.7 \pm 5.0$ \\
\hline \multicolumn{5}{|l|}{ UPCR category } \\
\hline$<0.5$ & $2(2)$ & 0 & $2(7)$ & 0 \\
\hline $0.5-3.0$ & $15(19)$ & $6(16)$ & $9(32)$ & 0 \\
\hline$>3.0$ & $64(79)$ & $32(84)$ & $17(61)$ & $15(100)$ \\
\hline $\mathrm{FPG}, \mathrm{mg} / \mathrm{dl}$ & $149 \pm 85$ & $156 \pm 81$ & $144 \pm 107$ & $141 \pm 55$ \\
\hline HbA1c, \% & $7.5 \pm 1.8$ & $7.8 \pm 1.9$ & $6.9 \pm 1.5$ & $7.4 \pm 1.7$ \\
\hline
\end{tabular}


Note: Data are presented as mean \pm standard deviation, median [interquartile range], or number (percent). Percentages may not total 100 due to rounding.

Abbreviations: DN, diabetic nephropathy; NDRD, non-diabetic renal disease; BMI, body mass index; DM, diabetes mellitus; DR, diabetic retinopathy; CVD, cardiovascular disease; eGFR, estimated glomerular filtration rate; Hb, hemoglobin; RBC, red blood cell; UPCR, urine protein creatinine ratio, FPG, fasting plasma glucose; HbA1c, hemoglobin A1c.

\section{Table 2 Pathological diagnosis of NDRD}


Pathological diagnosis

Total NDRD NDRD

$\mathrm{DN}+\mathrm{NDRD}$

\begin{tabular}{lccc} 
& $\mathbf{( n = 4 3 )}$ & $\mathbf{( n = 2 8 )}$ & $\mathbf{( n = 1 5 )}$ \\
\hline Primary FSGS & $6(14.0)$ & $6(21.4)$ & 0 \\
Primary membranous nephropathy & $6(14.0)$ & $4(14.3)$ & $2(13.3)$ \\
Pauci-immune glomerulonephritis & $5(11.6)$ & $4(14.3)$ & $1(6.7)$ \\
Lupus nephritis & $2(4.6)$ & $2(7.1)$ & 0 \\
IgA nephropathy & $5(11.6)$ & $3(10.7)$ & $2(13.3)$ \\
Post-infectious glomerulonephritis & $5(11.6)$ & $1(3.6)$ & $4(26.7)$ \\
Secondary FSGS & $6(14.0)$ & $3(10.7)$ & $3(20.0)$ \\
Hypertensive nephrosclerosis & $3(7.0)$ & $3(10.7)$ & 0 \\
Acute tubular necrosis & $4(9.3)$ & $2(7.1)$ & $2(13.3)$ \\
Acute interstitial nephritis & $2(4.7)$ & $2(7.1)$ & 0 \\
Thrombotic microangiopathy & $1(2.3)$ & $1(3.6)$ & 0 \\
Others & $1(2.3)$ & 0 & $1(6.7)$ \\
\hline
\end{tabular}

Note: Data are presented as number (percent). Percentages may not total 100 due to rounding.

Abbreviations: NDRD, non-diabetic renal disease; DN, diabetic nephropathy; FSGS, focal segmental glomerulosclerosis; IgA, immunoglobulin A.

Table 3 Univariable and multivariable binary logistic models to define the association of predictors in participants with any type of NDRD as compared to DN alone 


\begin{tabular}{lcccccc}
\hline \multirow{2}{*}{ Variables } & \multicolumn{3}{c}{ Univariable model } & \multicolumn{3}{c}{ Multivariable model $^{\mathrm{a}}$} \\
\cline { 2 - 6 } & OR & 95\% CI & $\boldsymbol{p}$ value & OR & 95\% CI & $\boldsymbol{p}$ value \\
\hline Age (per 1 year) & 1.02 & $0.99-1.06$ & 0.22 & & & \\
Male & 1.05 & $0.44-2.51$ & 0.92 & & & \\
BMI (per 1 kg/m²) & 1.03 & $0.95-1.11$ & 0.53 & & & 0.46 \\
Duration of DM <10 years & 2.57 & $1.04-6.31$ & 0.04 & 1.50 & $0.52-4.35$ & 0.02 \\
Absence of DR & 4.48 & $1.70-11.8$ & $<0.01$ & 3.72 & $1.28-10.8$ & \\
Hypertension & 1.39 & $0.36-5.31$ & 0.64 & & & \\
Established CVD & 1.43 & $0.40-5.11$ & 0.59 & & & \\
eGFR >30 ml/min/1.73m ${ }^{2}$ & 1.97 & $0.81-4.80$ & 0.14 & 2.31 & $0.73-7.34$ & 0.16 \\
Hemoglobin (per 1 g/dl) & 1.08 & $0.89-1.31$ & 0.46 & & & \\
Absence of hematuria & 1.81 & $0.66-4.96$ & 0.25 & & & \\
UPCR (per 1 mg/g) & 0.96 & $0.90-1.03$ & 0.27 & & & \\
HbA1c <8 \% & 2.47 & $0.88-6.88$ & 0.09 & 2.82 & $0.79-10.0$ & 0.11 \\
\hline
\end{tabular}

Note: Predictors in multivariable model were selected using $p$ value $<0.20$ in univariable model.

an the multivariable model (developed model), the equation is logodds (NDRD) $=$ $-1.75+0.41(\mathrm{DM}$ duration <10 years $)+0.84\left(\mathrm{eGFR}>30 \mathrm{ml} / \mathrm{min} / 1.73 \mathrm{~m}^{2}\right)+1.03$ $(\mathrm{HbA} 1 \mathrm{c}<8 \%)+1.31$ (absence of DR). Odd ratios are calculated from exponentiation of coefficients in the model.

Abbreviations: OR, odds ratio; CI, confidence interval; BMI, body mass index; DM, diabetes mellitus; DR, diabetic retinopathy; CVD, cardiovascular disease; eGFR, estimated glomerular filtration rate; HbA1c, hemoglobin A1c.

Table 4 The estimated probability being NDRD from developed model and optimismadjusted model according to clinical prediction score 


\begin{tabular}{ccc}
\cline { 2 - 3 } & Developed model $^{\mathrm{b}}$ & Optimism-adjusted model $^{\mathbf{c}}$ \\
\hline 0 & $14.8 \%$ & $21.1 \%$ \\
2 & $20.7 \%$ & $26.7 \%$ \\
3 & $31.1 \%$ & $35.8 \%$ \\
4 & $41.0 \%$ & $43.8 \%$ \\
5 & $53.1 \%$ & $53.2 \%$ \\
6 & $63.0 \%$ & $61.0 \%$ \\
7 & $72.2 \%$ & $68.4 \%$ \\
8 & $80.8 \%$ & $76.0 \%$ \\
\hline
\end{tabular}

aScore total is calculated from four predictors: score of 1 for "DM duration $<10$ years", score of 2 for "eGFR $>30 \mathrm{ml} / \mathrm{min} / 1.73 \mathrm{~m}^{2}$ " and "HbA1c $<8 \%$ ", and score of 3 for "absence of DR".

${ }^{\mathrm{b}}$ In developed model, the equation is logodds (NDRD) $=-1.75+0.41$ (DM duration $<10$ years $)+0.84\left(\right.$ eGFR $\left.>30 \mathrm{ml} / \mathrm{min} / 1.73 \mathrm{~m}^{2}\right)+1.03(\mathrm{HbA} 1 \mathrm{c}<8 \%)+1.31$ (absence of DR)

CIn optimism-adjusted model, optimism was calculated from bootstrapping model with the uniform shrinkage factor of 0.77 and the revised intercept of -1.32 . The equation is logodds $(\mathrm{NDRD})=-1.32+0.31(\mathrm{DM}$ duration $<10$ years $)+0.65(\mathrm{eGFR}$ $\left.>30 \mathrm{ml} / \mathrm{min} / 1.73 \mathrm{~m}^{2}\right)+0.80(\mathrm{HbA} 1 \mathrm{c}<8 \%)+1.02$ (absence of DR) Abbreviations: NDRD, non-diabetic renal disease; DM, diabetes mellitus; eGFR, estimated glomerular filtration rate; HbA1c, hemoglobin A1c; DR, diabetic retinopathy.

\section{Figures}




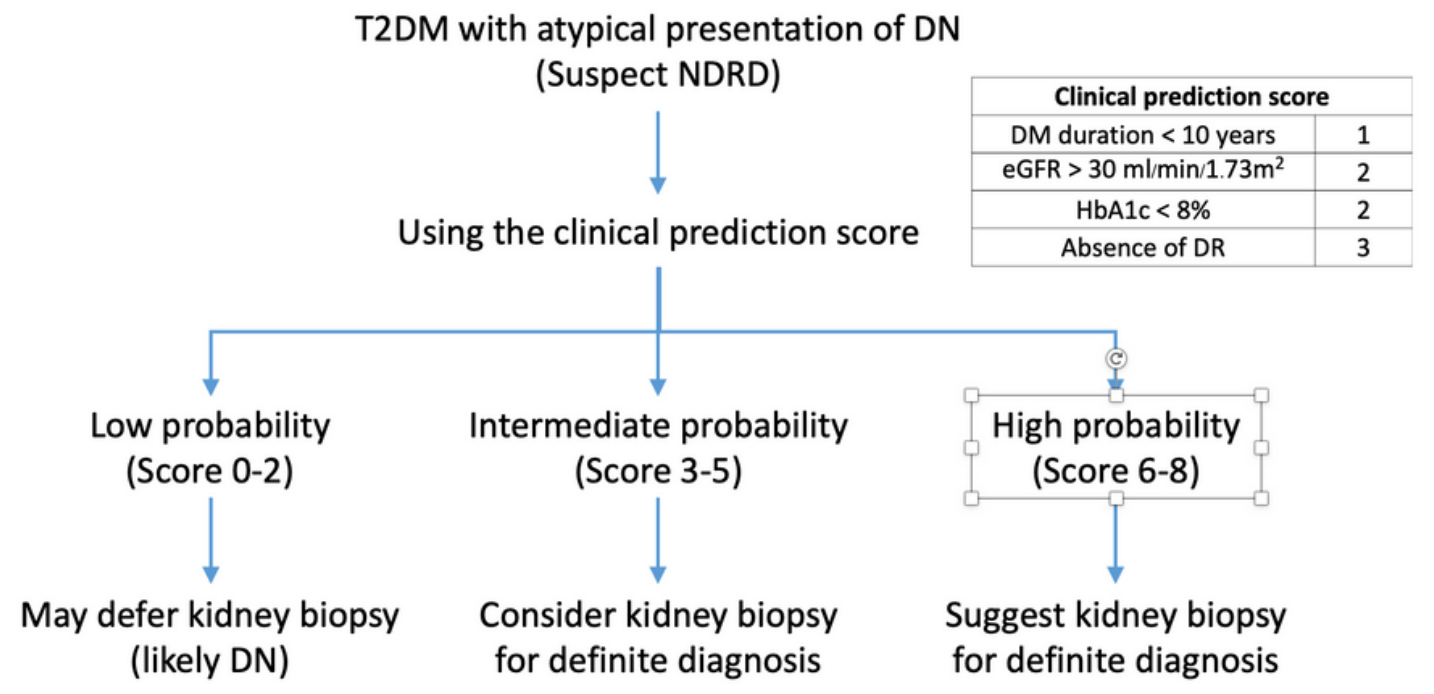

Figure 1

Proposed algorithm using the clinical prediction score A proposed algorithm for clinical use of the clinical prediction score, composed of four predictors and categorized into three groups. The low probability (score 0-2) of NDRD group means less likelihood of NDRD (or likely DN), which may defer kidney biopsy. The intermediate probability (score 3-5) and high probability for NDRD (6-8) suggests a kidney biopsy is necessary for definite diagnosis. Abbreviations: NDRD, non-diabetic renal disease; DN, diabetic nephropathy; DM, diabetes mellitus; eGFR, estimated glomerular filtration rate; $\mathrm{HbA1c}$, hemoglobin $\mathrm{A} 1 \mathrm{c}$; $\mathrm{DR}$, diabetic retinopathy. 